\title{
Robotic Surgery in Otolaryngology During the Covid-19 Pandemic: A Safer Approach?
}

\author{
Akriti Sharma $^{1} \cdot$ Rohit Bhardwaj ${ }^{2}$
}

Received: 17 June 2020/Accepted: 31 July 2020/Published online: 5 August 2020

(C) Association of Otolaryngologists of India 2020

\begin{abstract}
The COVID-19 outbreak was announced a pandemic by the World Health Organization in March 2020. The contagious nature of this virus to spread through droplets, puts the ENT surgeon at an added risk of exposure and infection, particularly while performing various aerosol generating procedures of the oral cavity and upper airway. While it is being recommended worldwide to postpone all elective surgeries to a later date, certain emergency procedures must be undertaken promptly but in a manner that minimizes the exposure of the surgeon and health care staff to the SARS-CoV-2. This article is meant to provide an insight into the possible role of robotic surgery in Otolaryngology and Head and Neck during the Covid-19 pandemic along with providing a brief review of its pros and cons.
\end{abstract}

Keywords COVID-19 - Otolaryngology ·

Robotic surgery $\cdot$ Pros $\cdot$ Cons

\section{Introduction}

Coronavirus disease 2019 (COVID-19) is caused by the severe acute respiratory syndrome coronavirus 2 (SARSCoV-2) [1]. The contagious nature of SARS CoV-2 puts

Rohit Bhardwaj

rohitandlife@gmail.com

1 Department of Otorhinolaryngology, SGT Medical College, Hospital \& Research Institute, Village Budhera, Gurugram, Haryana 122505, India

2 Department of Otorhinolaryngology, Safdarjung Hospital and Vardhman Mahavir Medical College, Ansari Nagar, New Delhi 110029, India the ENT surgeon at a high risk of being infected with the virus $[2,3]$ particularly while performing aerosol generated procedures.

Procedures of the nose, oral cavity, larynx, pharynx and trachea have a high risk of generating aerosols [4]. While we endorse the recommendation that all elective cases should be postponed during this time and that only emergency procedures be carried out [5], this must be done in a manner that limits the risk of exposure of the surgeon and the health care staff to Covid-19. In the current scenario, when social distancing is considered to play a key role in preventing the spread of Covid-19, Robotic surgery could prove to be the surgeon's choice for management of patients requiring surgical intervention. The procedure is carried out by a surgeon sitting at a console in the operating room, but outside the sterile field, directing and controlling the motions of the robotic arms, thus maintaining complete control over the surgery with the only difference being that the control is indirect and exercised from an increased distance.

Though the application of Robotic surgery in the field of ENT is limited, it could play a significant role in the management of head and neck malignancies. In the current scenario, Robot Assisted Surgery, wherever available and indicated, can prove to be beneficial in effective management of essential ear, nose, throat, and head and neck conditions that cannot be postponed to a later date while minimizing the exposure of the operating surgeon reducing the risk of cross infection among people working in OT, to the aerosols generated during the procedure.

However, the present technology could have certain limitations too. An important point to take note of is that the surgeon sits at the console and is away from the patient's mouth but the assistant is at the head of the bed and in close proximity to the patient. The assistant is quite 
exposed to secretions and electrocautery fumes. Therefore, the benefit of being away from the patient may not apply to the entire team, but the majority of health care staff could still be protected. We believe that the pros and cons of every procedure must be evaluated thoroughly before undertaking it and complete knowledge of the precautions that need to be taken while performing a surgery is a must. This article provides a comprehensive review of the advantages and disadvantages of carrying out Robotic surgery for the management of Ear, Nose, throat and head and neck cases during the Covid-19 pandemic and the precautions that are required to be kept in mind while performing the surgery.

\section{Indications of Robot Assisted Surgery in Cases of Otolaryngology and Head and Neck}

It is being recommended worldwide that all elective surgeries be postponed to a later date, certain emergent conditions requiring prompt intervention need to be triaged. During the Covid-19 pandemic, the levels of surgical prioritization have been detailed by the Royal college of physicians and surgeons Glasgow in novel studies [6].

Robot assisted surgery in ENT can be classified into Transoral Robotic Surgery (TORS) for lesions in the oral cavity oropharynx, larynx and hypopharynx and Remote access surgery done through a Retro Auricular Hairline Incision (RAHI) for removal of neck masses without leaving a scar in the anterior aspect of neck [7]. Keeping this classification in mind, the triage guidelines for the current scenario [6] and the applications of robotic surgery for management of ear, nose, throat, head and neck

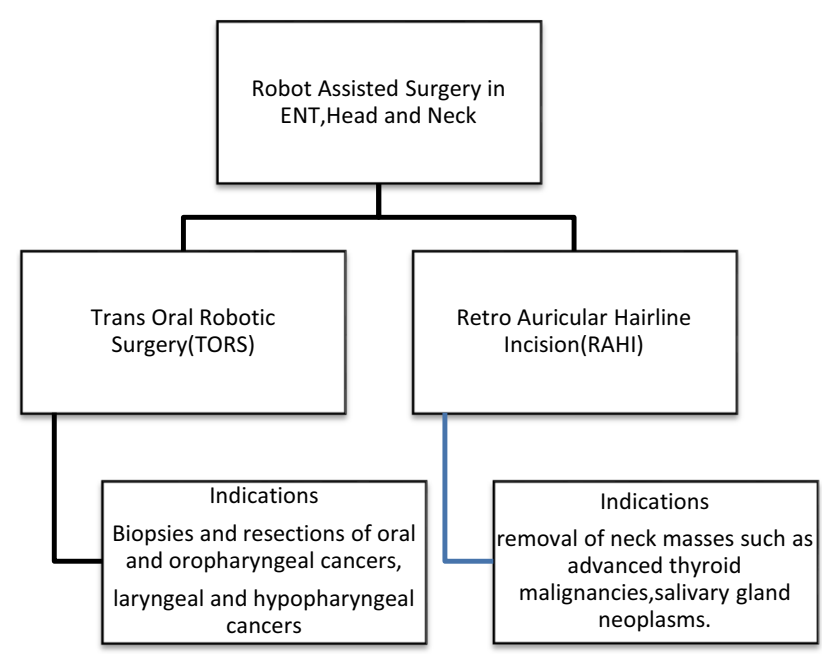

Fig. 1 Depicts classification and indications of robot assisted surgery in ENT and Head \& neck during the Covid-19 pandemic pathologies [8], Fig. 1 depicts the indications of robotic surgery during the Covid-19 outbreak.

Though conservative management and postponement of non-urgent surgeries is recommended during the Covid-19 pandemic, surgical intervention, if needed for the treatment of malignancies must not be postponed beyond a limit. If patients with head and neck malignancies present at an early stage where resection of the tumor is feasible, they can expect a good outcome after the surgery and appropriate adjuvant treatment [9]. A delay in surgery of such a patient could lead to progression of the disease and ultimately could worsen the prognosis of the condition. A triage of cancer cases is hence mandatory. The American College of Surgeons (ACS) has given guidelines for triage of cancer surgeries during this pandemic [10]. In our view the decision of surgery should depend on the stage of tumor and its characteristics. While one might consider postponing the surgery in slowly progressive tumours in the early stage some tumours may warrant prompt resection and adjuvant treatment for better prognosis.

While the use of Robotic Surgery is limited in transnasal and otological procedures because of instrument size and functionality [11], its application could prove to be beneficial for the management of head and neck neoplasms and could have various advantages over open surgeries during the Covid-19 outbreak.However, this might prove to be an expensive treatment alternative and the equipment required for it may not be available in many centers.

While the advantages and disadvantages of robotic surgery in ENT have been documented in studies earlier [8], assessing its application while dealing with a contagious virus like SARS-CoV-2 is the need of the hour.

\section{PROS}

The primary advantage of Robotic surgery during the Covid-19 pandemic is that it can be performed by maintaining a distance not only between the patient and the health care workers but also between the surgeon and other operating staff, thereby minimizing the risk of transmission of virus between health care workers who otherwise would be in closer contact with each other and the patients during conventional, open procedures [12].Transoral Robotic Surgery which has a major role to play in the management of head and neck malignancies, avoids procedures like mandibulotomy and reduces the need for tracheostomy both of which are procedures that generate aerosols in large quantities [11].Another major advantage that we can derive by use of Robot assisted surgery during this time include minimizing direct exposure of a majority of the healthcare personnel to body fluids, smoke, gases and aerosols generated in a surgical procedure. In addition to the above 
factors, Robotic surgery reduces the duration of stay of a patient in a hospital. Hence in accordance with novel studies [13] we are of the opinion that in the present scenario, benefits of robotic surgery far outweigh those of open procedures.

\section{CONS}

Robot Assisted Surgery in ENT has its own limitations which have been enumerated in studies earlier [8]. However the pitfalls of the procedure must be freshly evaluated before considering it as an important treatment modality during the Covid-19 Pandemic. The most important factors to take note of are the expensive equipment of surgery and its lack of availability in many treatment centers. The lack of experienced and trained personnel for carrying out the procedure may also pose a challenge. However, in centers where there is availability of the equipment as well as trained staff, Robot Assisted procedures can successfully be carried out to manage life threatening conditions in the current scenario with minimal exposure to the staff in the Operating Room..

Another important consideration is that the smoke and gases released during surgeries are potentially hazardous and some studies report the presence of viruses in smoke generated during surgical procedures [14]. The presence of a safe and effective method to vent smoke and gas generated during the surgery is hence essential.

\section{Recommendations for Robot Assisted Surgery During the Covid-19 Pandemic}

- The importance of triage cannot be undermined during the Covid-19 outbreak and we strongly believe that the first step towards patient management during the Covid19 pandemic should be prioritization and proper decision making keeping in mind that while prompt intervention must be carried out for all emergency cases, all precautions must be taken to minimize the exposure to the health care staff. Guidelines for the triage of ENT procedures must be followed sincerely, though we believe that the decision to operate upon a tumor could differ from case to case and should be the surgeon's call [6].

- We advocate pre-operative screening of all patients for Covid-19; $48 \mathrm{~h}$ prior to undergoing surgery. In centers where this is not possible, all patients must be considered Covid suspects and necessary precautions should be taken accordingly.

- Though cumbersome, the use of Personal protective equipment(PPE) and N95 masks with goggles is recommended to be worn by all OT staff while doing the robotic surgery. For the Personal protective equipment (PPE), use of highest protection level III is recommended for bedside assistant and level II for console surgeon [13].

- The time taken by the staff in docking and undocking of robotic arms should be minimum and hence only trained staff must be present in the Operation theatre.

- In addition to these, we recommend the use of instruments with integrated suctions along with limiting the number of assistants and trainees in the operating theatre.

- The Operation theaters must be equipped with an excellent ventilation system designed to facilitate evacuation of smoke, gases and aerosols effectively.

- We advocate the use of coblator over electrocautery for the purpose of hemostasis in surgery, wherever applicable.This would help in reducing smoke generation during the procedure.

- Since the viruses present in aerosols have a tendency to settle on metal surfaces, the pre and postoperative decontamination of the equipment of Robot Assisted Surgery could prove to be time consuming and challenging in the current scenario. The use of disinfectants for sterilization of all robotic surgery equipment must be made mandatory and fumigation of OT should be ensured after every procedure.

\section{Conclusion}

Based on a thorough reading of literature on the various recommendations being followed for surgeons worldwide during the Covid-19 Pandemic, we can state with conviction that any surgical procedure being undertaken in the current scenario should be done in a manner that ensures successful patient management along with minimal exposure to the health care staff. Robotic surgery is typically done in a manner that prevents direct contact of the surgeon with the patient and the health care staff. Robot Assisted Surgery if available, offers various benefits over open procedures in the current scenario and hence is the need of the hour. In accordance with certain studies [13] we would suggest that in the coming months, Robotic Surgery could prove to be a safer and more effective alternative option to open procedures with the view of minimizing exposure to SARS-CoV-2 provided all precautions, for the safety of Health care staff, along with measures of smoke evacuation and disinfection of equipment are meticulously undertaken.

Funding No funding was received. 


\section{Compliance with Ethical Standards}

Conflict of interest The authors declare that there was no conflict of interest.

\section{References}

1. del Rio C, Malani P (2020) COVID-19-New insights on a rapidly changing epidemic. JAMA 323(14):1339. https://doi.org/ 10.1001/jama.2020.3072

2. Chang D, Xu H, Rebaza A, Sharma L, Dela CC (2020) Protecting health-care workers from subclinical coronavirus infection. Lancet Respir Med 8(3):e13. https://doi.org/10.1016/s2213-2600 (20)30066-7

3. 404. BNN. https://www.bnnbloomberg.ca/why-surgeons-don-twant-to-operate-right-now-1.1411294. Published 2020. Accessed April 22, 2020. Published 2020. Accessed May 4, 2020.

4. Lu D, Wang H, Yu R, Yang H, Zhao Y (2020) Integrated infection control strategy to minimize nosocomial infection of coronavirus disease 2019 among ENT healthcare workers. J Hosp Infect 104(4):454-455. https://doi.org/10.1016/j.jhin.2020.02.018

5. Collaborative C (2020) Global guidance for surgical care during the COVID-19 pandemic. Br J Surg. https://doi.org/10.1002/ bjs. 11646

6. England.nhs.uk.https://www.england.nhs.uk/coronavirus/wp-cont ent/uploads/sites/52/2020/03/C0221-specialty-guide-surgical-pri oritisation-v1.pdf. Published 2020. Accessed 4 May 2020

7. Venkatakarthikeyan VC (2019) Robotics in ent head and neck surgery. Up State J Otolaryngol Head Neck Surg 7:15-20. https://doi.org/10.36611/upjohns/19.3
8. Oliveira C, Nguyen H, Ferraz A, Watters K, Rosman B, Rahbar R (2012) Robotic surgery in otolaryngology and head and neck surgery: a review. Minim Invasive Surg 2012:1-11. https://doi.org/ $10.1155 / 2012 / 286563$

9. Hamoir M, Holvoet E, Ambroise J, Lengelé B, Schmitz S (2017) Salvage surgery in recurrent head and neck squamous cell carcinoma: oncologic outcome and predictors of disease free survival. Oral Oncol 67:1-9. https://doi.org/10.1016/j.oraloncology. 2017.01.008

10. COVID-19 Guidelines for Triage of Cancer Surgery Patients. American College of Surgeons. https://www.facs.org/covid-19/ clinical-guidance/elective-case/cancer-surgery. Published 2020. Accessed May 9, 2020.

11. Rinaldi V, Pagani D, Torretta S, Pignataro L (2013) Transoral robotic surgery in the management of head and neck tumours. Ecancermedicalscience. https://doi.org/10.3332/ecancer.2013.359

12. Vigneswaran Y, Prachand V, Posner M, Matthews J, Hussain M (2020) What is the appropriate use of laparoscopy over open procedures in the current COVID-19 climate? J Gastrointest Surg. https://doi.org/10.1007/s11605-020-04592-9

13. Kimmig R, Verheijen R, Rudnicki M (2020) Robot assisted surgery during the COVID-19 pandemic, especially for gynecological cancer: a statement of the society of european robotic gynaecological surgery (SERGS). J GynecolOncol. https://doi.org/10.3802/jgo.2020.31.e59

14. Kwak H, Kim S, Seo Y, Song K (2016) Detecting hepatitis B virus in surgical smoke emitted during laparoscopic surgery . Occup Environ Med.https://doi.org/10.1136/oemed-2016-103724

Publisher's Note Springer Nature remains neutral with regard to jurisdictional claims in published maps and institutional affiliations. 